Precessi on drift reversal and rapi d transport of $t$ rapped energet i c parti cles due to an energet i c parti cle driven instability in the Large Hel i cal Devi ce

\begin{tabular}{|l|l|}
\hline $\begin{array}{l}\text { jour nal or } \\
\text { publ i cat i on } \mathrm{titl} \text { e }\end{array}$ & Physi cs of Pl asmas \\
\hline vol une & 28 \\
\hline page $\mathrm{r}$ ange & 080701 \\
\hline year & $2021-08$ 05 \\
\hline URL & ht t p: //hdl . handl e. net /10655/00012675 \\
\hline
\end{tabular}




\title{
Precession drift reversal and rapid transport of trapped energetic particles due to an energetic-particle driven instability in the Large Helical Device
}

\author{
M. Idouakass, ${ }^{1,}$ a) Y. Todo, ${ }^{1}$ H. Wang, ${ }^{1}$ J. Wang, ${ }^{1}$ R. Seki, ${ }^{1,2}$ and M. Sato ${ }^{1}$ \\ ${ }^{1)}$ National Institute for Fusion Science, National Institute of Natural Sciences, 322-6 Oroshi-cho, Toki, \\ Gifu 509-5292, Japan \\ ${ }^{2)}$ Department of Fusion Science, SOKENDAI (The Graduate University for Advanced Studies), Toki 509-5292, \\ Japan
}

(Dated: 18 November 2021)

The energetic-particle transport by a magnetohydrodynamic (MHD) instability driven by helically-trapped energetic particles is studied for a high-performance Large Helical Device plasma with kinetic-MHD hybrid simulations. It is observed in the simulation that an MHD mode with poloidal/toroidal mode numbers $m / n=2 / 1$ driven by helically-trapped energetic particles causes a significant redistribution of perpendicular energetic-particle pressure profile. The frequency of the MHD mode decreases rapidly at the saturation of the instability and changes the sign, which indicates the reversal of the mode propagation direction. It is found that the helically-trapped energetic particles which are interacting strongly with the MHD mode change the precession drift direction at the same time as the reversal of the MHD mode propagation direction. The helically-trapped energetic particles with the precession drift reversal are transported rapidly in the radially outward direction before the original precession drift direction is recovered. The precession drift reversal and the outward transport is caused by the interaction with the electric field of the MHD mode. The vast majority of trapped energetic particles which interact strongly with the MHD mode experience the precession drift reversal, leading to the significant redistribution of perpendicular energetic-particle pressure profile.

Energetic particle (EP) physics is a crucial component in the understanding of plasmas in present and future magnetic confinement fusion devices. In this context, energetic particles refer to particles that have kinetic energy much higher than the average particle thermal energy. They can be produced by external heating mechanisms such as neutral beam injection (NBI) and wave-particle resonance (e.g. ICRH/ECRH), or they are the fusion produced alpha particles in the case of $\mathrm{D}-\mathrm{T}$ fusion.

In order to maintain favorable conditions for fusion, the energetic particles need to remain confined inside the plasma core long enough to deposit most of their energy onto the thermal particle population. Due to their high energy, EPs can have a strong influence on the global behavior of the plasma, by for example destabilizing magnetohydrodynamic (MHD) modes in the devices, which can lead to losses of energetic particles, thus reducing their ability to maintain the high plasma temperature required for fusion. This phenomenon is present in different configurations, with various different modes, for example the fishbone instability ${ }^{1-3}$ in tokamaks, or toroidal Alfvén eigenmodes destabilized by energetic particles in tokamaks $^{4-11}$ as well as in stellarators ${ }^{12,13}$.

A critical MHD instability driven by energetic particles has been recently observed in the Large Helical Device (LHD): the energetic particle driven interchange mode (EIC $)^{14-19}$. It is an $m / n=1 / 1$ resistive interchange mode destabilized by energetic particles generated by perpendicular NBI, where $m$ and $n$ are the poloidal and toroidal mode numbers respectively. It was observed that

\footnotetext{
a)idouakass.malik@nifs.ac.jp
}

the EIC ejects energetic particles from the core plasma, thus reducing the neutron yield in deuterium experiments. One of its characteristics is the down-chirping of the frequency during each burst, a similar behavior to that of the fishbone mode in tokamaks.

The observations of this mode motivate us to study MHD instabilities driven by a population of majority trapped energetic particles (as expected from the perpendicular NBI) in the LHD. This study is conducted using the kinetic-MHD hybrid code MEGA ${ }^{20,21}$, which allows to treat the bulk plasma as a fluid using the MHD equations, and to describe the energetic particle dynamics using the drift-kinetic model. The use of such a hybrid model allows to study non-Maxwellian distribution functions and wave-particle resonances for the energetic particles. MEGA has been successfully used to study wave-particle interactions in multiple tokamaks ${ }^{22-26}$ as well as the $\mathrm{LHD}^{27-30}$. In this work, an $m / n=1 / 1 \mathrm{EIC}$ mode is not observed, which may be due to the fixed boundary condition chosen or other effects that will be investigated in a later study. This letter is instead focused on the destabilization of an $m / n=2 / 1$ mode, closer to the plasma center than the EIC, by an anisotropic population of energetic particles in a high-performance deuterium LHD plasma.

The case discussed in this letter uses an equilibrium calculated with the HINT code ${ }^{31,32}$ based on the LHD shot \#135730 at $4.843 \mathrm{~s}$, which has a central electron temperature $T_{e}=3.5 \mathrm{keV}$, a central ion temperature $T_{i}=10 \mathrm{keV}$ and a central electron density $n_{e}=1.766 \times$ $10^{19} \mathrm{~m}^{-3}$. The central magnetic field in this equilibrium is $B_{0}=2.85 \mathrm{~T}$. This equilibrium tends to be favorable for the destabilization of an $m / n=2 / 1$ mode centered around the $\iota=0.5$ surface. Therefore, a population 
of energetic particles with a pitch angle consistent with perpendicular NBI and a simplified pressure profile well suited to study this mode is added. The whole device is simulated in cylindrical geometry $(R, \varphi, Z)$ with a resolution of $(128,640,128)$. The energetic particles are simulated using a $\delta f$ particle-in-cell method. In the present simulation, approximately $8 \times 10^{7}$ particles were used.

The energetic particle population has a slowing-down distribution in velocity space, and a Gaussian profile in pitch-angle space. The energetic particle distribution reads:

$$
\begin{array}{r}
f(v, \Lambda)=\frac{F_{0}}{v^{3}+v_{\text {crit }}^{3}} \times 0.5 \times \operatorname{erfc}\left(\frac{v-v_{\text {birth }}}{\Delta_{v}}\right) \times \\
\exp \left(\frac{-\left(\Lambda-\Lambda_{0}\right)^{2}}{\Delta_{\Lambda}^{2}}\right)
\end{array}
$$

where $v$ is the particle velocity, $F_{0}$ is a normalization factor, $v_{c r i t}$ is the slowing down distribution's critical velocity, $v_{b i r t h}$ is the injection velocity, $\Delta_{v}$ represents the distribution function cut-off steepness in velocity direction (linked to the injection beam width), $\Lambda=\mu B_{0} / E_{k i n}$ is the pitch-angle variable (with $\mu$ the magnetic moment, $E_{k i n}$ is the particle kinetic energy), $\Lambda_{0}$ is the center of the Gaussian in the $\Lambda$ direction, and $\Delta_{\Lambda}$ is the standard variance. In this context, a higher (lower) value of $\Lambda_{0}$ implies a higher (lower) proportion of trapped particles. The spatial profile of energetic particle pressure, peaked on the plasma center, reads:

$$
\beta_{h}(r)=0.5 \times \beta_{h, 0} \times \operatorname{erfc}\left(\frac{r-r_{0}}{r_{\text {scale }}}\right)
$$

with $\beta_{h, 0}$ the EP pressure normalized by the magnetic pressure at the plasma center, and $r_{0}$ and $r_{\text {scale }}$ the center and scale of pressure radial fall-off normalized by the plasma minor radius. The perpendicular and parallel components of $\beta_{h}$ are calculated using the distribution function.

The case studied in this letter uses an energetic deuterium population with $\beta_{h, 0}=0.01, r_{0}=0.57$ (corresponding to the $\iota=0.5$ surface), $r_{\text {scale }}=0.2$. The energetic particles have a birth velocity $v_{\text {birth }}=0.229$ (corresponding to $60 \mathrm{keV}$ injection energy), and a critical velocity $v_{\text {crit }}=0.1985$ and $\Delta_{v}=0.023$, normalized by the Alfvén velocity at the plasma center $v_{A}=1.05 \times 10^{7} \mathrm{~m} / \mathrm{s}$. The pitch-angle distribution is defined with the parameters $\Lambda_{0}=1$ and $\Delta_{\Lambda}=0.3$. This corresponds to a high proportion of trapped energetic particles, consistent with the perpendicular NBI. For these parameters, the MHD instability with the dominant harmonic $m / n=2 / 1$ is destabilized by energetic particles with a high growth rate $\gamma \approx 4.4 \times 10^{4} \mathrm{~s}^{-1}$ and a frequency $\mathrm{f}_{0} \approx 17 \mathrm{kHz}$. Despite a number of differences in simulation conditions, this mode shows similarities with $m / n=2 / 1$ modes simulated with FAR3D ${ }^{18,19}$, specifically when the negative EP pressure radial gradient is around the $\iota=0.5$ surface, with the remaining difference that the mode profile observed here has a much larger radial extension.
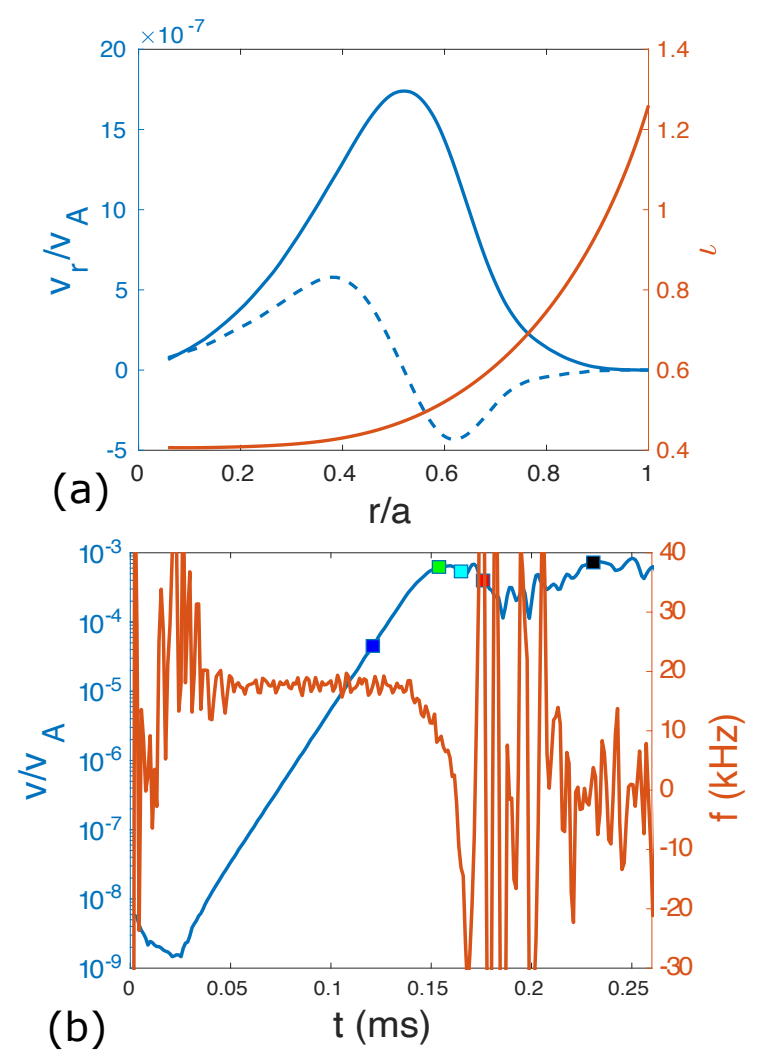

FIG. 1. (a) Blue: radial MHD velocity $v_{r} / v_{A}$ profile with $m / n=2 / 1$ during the linear phase, solid line is the cosine part and the dashed line is the sine part. Orange: rotational transform ( $\iota$ ) profile. (b) Time evolution of $v_{r} / v_{A}$ in blue and of the mode frequency in orange. The colored squares correspond to the different times of the snapshots presented in Fig. 2.

In this letter, we will focus on the nonlinear behavior of the instability. The radial MHD velocity profile of the instability is shown in Fig. 1(a) with the rotational transform $(\iota)$ profile. The time evolution of the amplitude and the frequency of the radial MHD velocity at the spatial peak location is shown in Fig. 1(b). We can see in Fig. 1(b) that the mode, after an exponential growth during the linear phase, saturates with a rather high radial MHD velocity value of approximately $v_{r} \approx 10^{-3} v_{A}$. At the same time, after being constant during the linear phase, the mode frequency drops very rapidly at the saturation, enough to become negative. The frequency sign reversal is a defining characteristic of this instability, and it should be noted it occurs rapidly, within less than a mode period. Then during the rest of the nonlinear phase, the mode frequency becomes too noisy to be read properly.

One of the most important characteristics of this mode is that it generates a very strong energetic particle redistribution in a short time during the saturation phase. The radial profiles of the energetic particle pressure, both parallel and perpendicular, are shown for various times after the saturation in Fig. 2. For the perpendicular pres- 


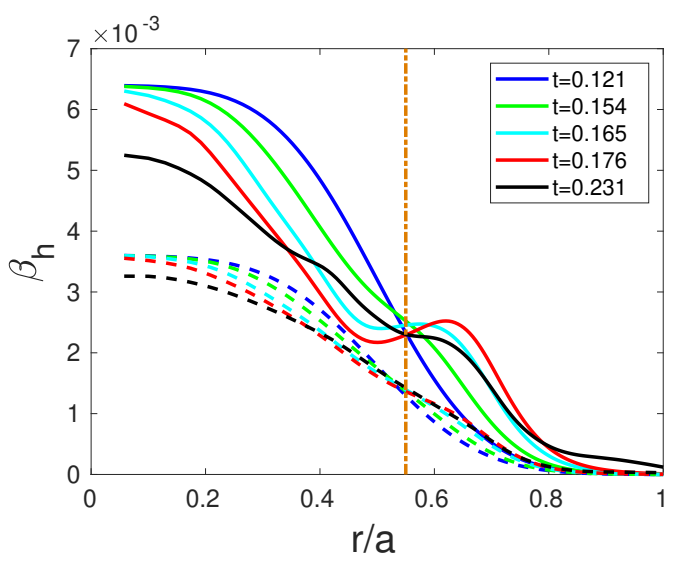

FIG. 2. Snapshots of the energetic particle pressure at different times. In solid line is the perpendicular pressure and in dashed line is the parallel pressure. Colors: blue: $t=0.121$ ms, green: $t=0.154 \mathrm{~ms}$, cyan: $t=0.165 \mathrm{~ms}$, red: $t=0.176$ ms, black: $t=0.231 \mathrm{~ms}$. The vertical dot-dashed line corresponds to the $\iota=0.5$ radius.

sure, the gradient starts to flatten (green curve) around the $\iota=0.5$ surface radius, then the gradient is reversed at this position (cyan and red curves), before the gradient becomes negative again throughout the radius, with a decreasing central pressure. While the parallel EP pressure also flattens at the resonance position and decreases in the center, the variation is comparatively smaller, and no pressure gradient reversal is observed.

We have analyzed the precession drift frequency of energetic particles in poloidal and toroidal angles $\left(\mathrm{f}_{\varphi}\right.$ and $\mathrm{f}_{\theta}$ ) averaged over the bounce motion. The distribution of energetic particles which are strongly interacting with the MHD mode is shown in $\left(\mathrm{f}_{\varphi}, \mathrm{f}_{\theta}\right)$ space in Fig. 3. All the particles plotted are trapped particles in the helical ripple of the magnetic field in LHD. We can see in Fig. 3 (a) their distribution during the linear phase. It can be noted that all the trapped particles roughly fall on a line having a $\mathrm{f}_{\theta}=-5 \times \mathrm{f}_{\varphi}$ dependency. This is due to the magnetic structure of LHD, where the trapped particles follow the helical shape of the machine, staying in the region of lower magnetic field. Second, we can see that the precession drift direction of all particles is the same, with positive $\theta$-direction, and negative $\varphi$-direction, and that there is a range of frequencies, going from close to zero to approximately $8 \mathrm{kHz}$ in $\theta$-direction. Finally, it is shown that the particles that interact most strongly with the mode tend to have a smaller range of $\mathrm{f}_{\theta}$ centered around $\mathrm{f}_{\theta}=2 \mathrm{kHz}$.

What is interesting for this instability is that, during the nonlinear phase, there is a strong redistribution in precession drift frequency, which still follows the expected line with $\mathrm{f}_{\theta}=-5 \times \mathrm{f}_{\varphi}$, where for a significant number of them, $\mathrm{f}_{\theta}$ becomes negative. This can be seen in Fig. 3(b) which represents the position of trapped particles in $\left(\mathrm{f}_{\varphi}, \mathrm{f}_{\theta}\right)$-space in the nonlinear phase.

The precession drift reversal starts at the same time as
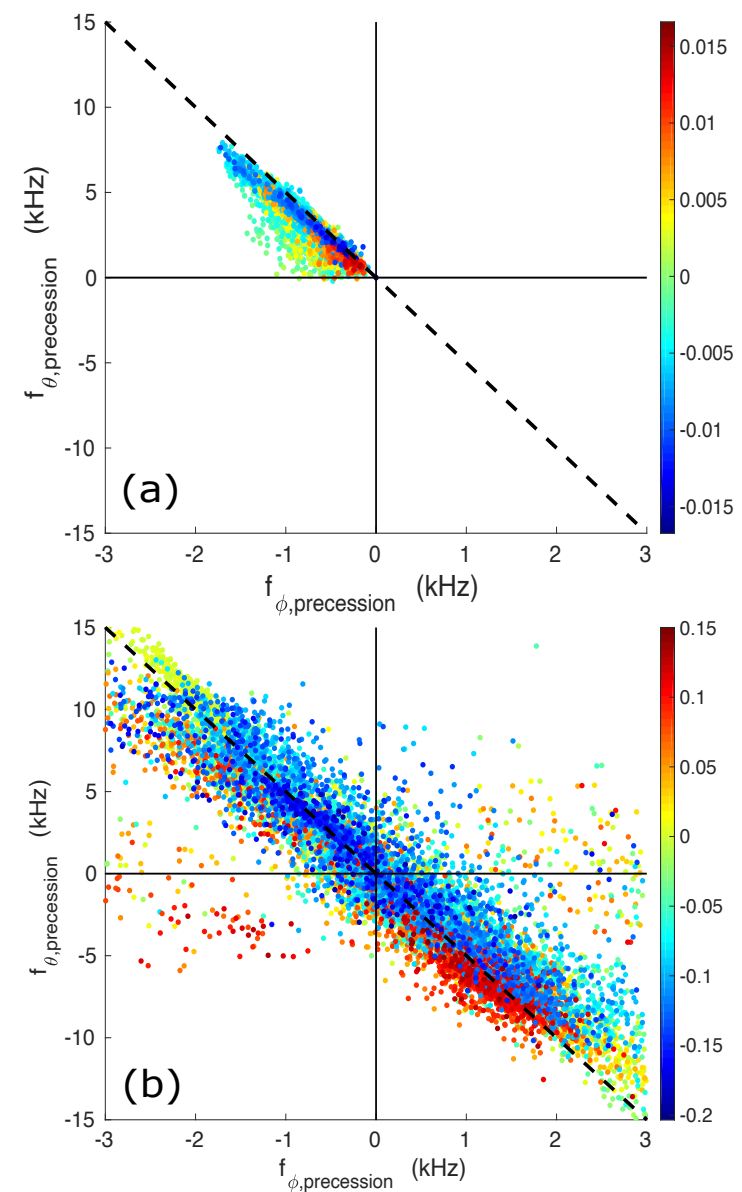

FIG. 3. Distribution of trapped energetic particles in the precession frequency $\left(\mathrm{f}_{\varphi}, \mathrm{f}_{\theta}\right)$-space. The color indicates the value of $\delta f$, which denotes the strength of interaction with the mode. (a) During the linear phase $(t=0.13 \mathrm{~ms})$. (b) During the nonlinear phase $(t=0.19 \mathrm{~ms})$

the mode frequency begins chirping. During the nonlinear saturation phase, an increasing number of helicallytrapped energetic particles change their precession drift direction. This phenomenon culminates at approximately $t=0.2 \mathrm{~ms}$ in the time evolution shown in Fig. 1 when roughly $40 \%$ of trapped particles that interact strongly with the mode have their precession drift in the reversed direction which we can see in Fig. 4(a). Figure 4(a) also shows that, while the number of particles with the precession drift reversal starts increasing at the same time as the mode frequency starts chirping, a significant number of particles change their precession drift direction with a delay. Overall, during the nonlinear phase, almost all particles that remain helically-trapped and interact strongly with the mode have had their precession drift reversal before going back to their original precession drift direction at a higher radius.

An example is given of a typical particle trajectory in Fig. 5, where we see the trapped particle's evolution in three phases. The first phase, shown in blue is the trajectory during the end of the linear phase, with 

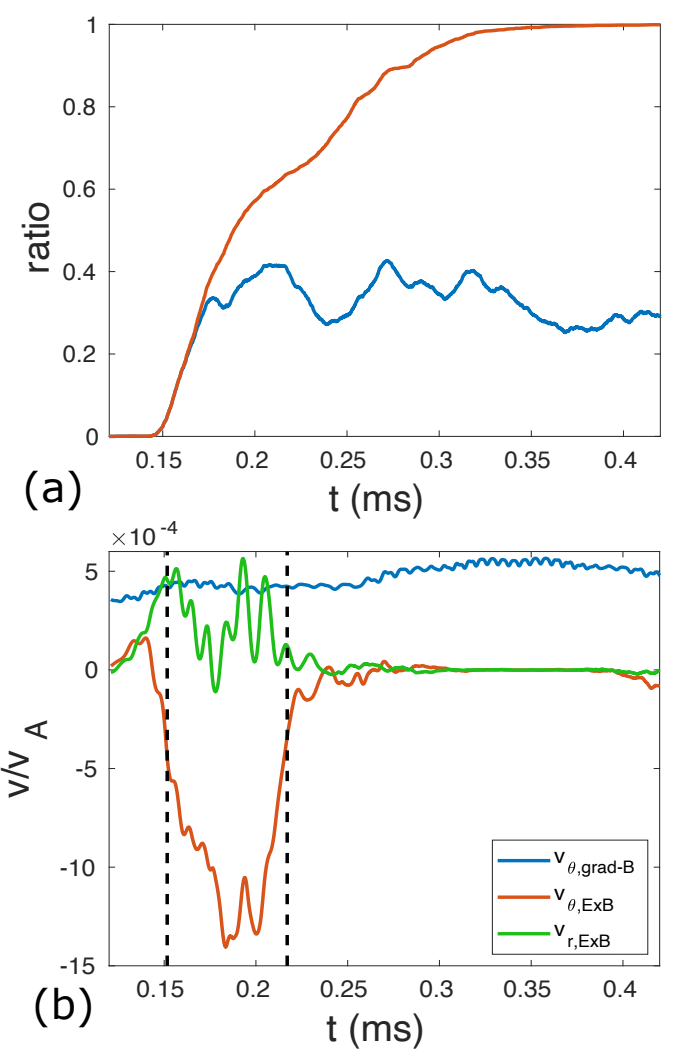

FIG. 4. (a) Ratios of the particles which have ever had the precession drift reversal (orange) and the particles which have the precession drift reversal at the moment (blue) to the helically-trapped particles that interact strongly with the mode. (b) Blue: averaged $\theta$-direction grad-B drift velocity of the particle whose trajectory is shown in Fig. 5. Red: $\theta$-direction $\mathrm{E} \times \mathrm{B}$ drift velocity. Green: radial $\mathrm{E} \times \mathrm{B}$ drift velocity. In the time period between the two vertical dashed lines, the precession drift direction is reversed.

the perturbation small enough that it is equivalent to an equilibrium trajectory. In the second phase, shown in green, the precession drift direction is reversed, and the particle travels further from the plasma center. Finally, in the third phase, the particle returns to the classical direction, and its trajectory is located at a higher radius.

We have analyzed contributions from grad-B, curvature, and $\mathrm{E} \times \mathrm{B}$ drifts averaged in one bounce motion for the particle trajectory shown in Fig. 5. For trapped particles, curvature drift is smaller than grad-B drift. Figure 4(b) shows the time evolution of $\theta$-direction grad-B drift velocity, $\theta$-direction $\mathrm{E} \times \mathrm{B}$ drift velocity, and radial $\mathrm{E} \times \mathrm{B}$ drift velocity. We see in the figure that $\theta$-direction grad$\mathrm{B}$ drift velocity is always positive, which indicates that grad-B drift causes the original precession drift motion in positive $\theta$ direction. On the other hand, $\theta$-direction $\mathrm{E} \times \mathrm{B}$ drift velocity is negative and the absolute value exceeds the grad-B drift velocity during the time period of precession drift reversal represented by the two vertical dashed lines. This clearly indicates that the $\mathrm{E} \times \mathrm{B}$ drift causes
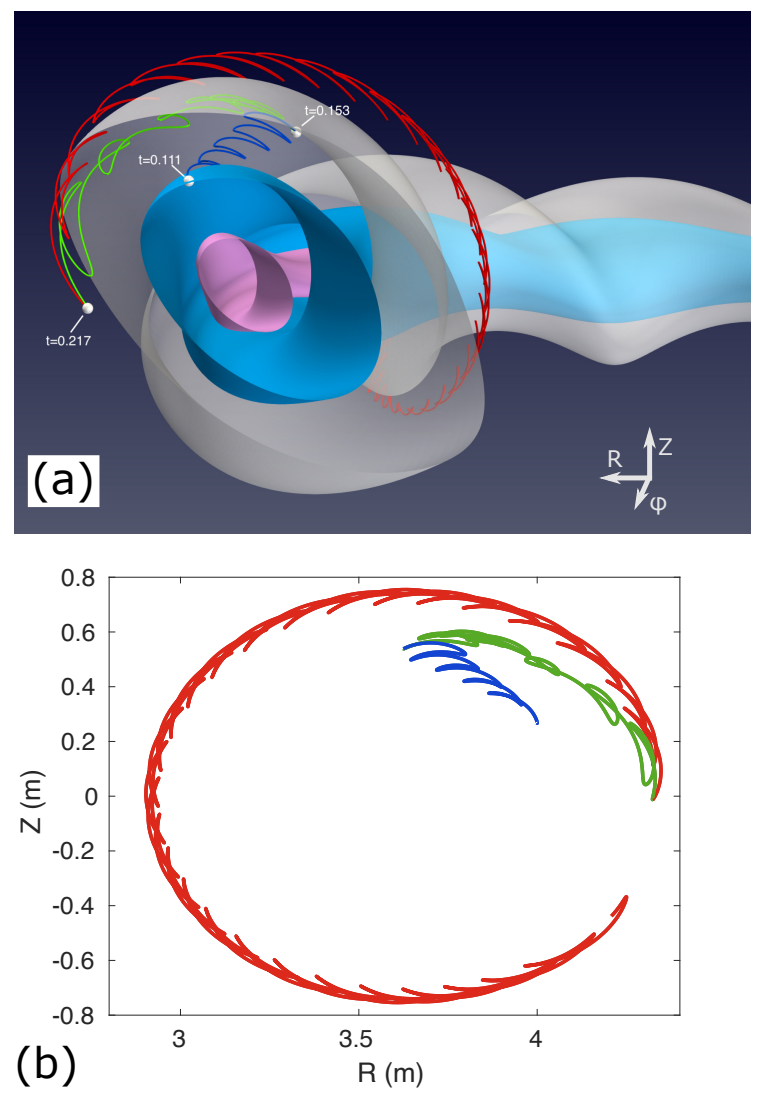

FIG. 5. (a) 3-dimensional representation of a typical particle trajectory, with magnetic flux surfaces shown for context. Magnetic flux surfaces radii: pink is $r / a=0.2$, blue is $r / a=0.5$, gray is $r / a=0.85$. The colored line is the particle trajectory. Blue: trajectory from $t=0.11 \mathrm{~ms}$ to $t=0.153 \mathrm{~ms}$. Green: trajectory from $t=0.153 \mathrm{~ms}$ to $t=0.216 \mathrm{~ms}$. Red: trajectory from $t=0.216 \mathrm{~ms}$ to $t=0.44 \mathrm{~ms}$. (b) Poloidal projection of the particle's trajectory in the same colors as in the $3 \mathrm{D}$ figure.

the precession drift reversal, which fits well within the theoretical framework provided in Ref. ${ }^{11,33}$. We see also in the figure that the radial $\mathrm{E} \times \mathrm{B}$ drift velocity is positive during the precession drift reversal leading to the radially outward transport of the particle. The interaction with the electric field lasts long enough for the trapped particle to be pushed to a significantly higher radius, before the $\mathrm{E} \times \mathrm{B}$ drift decreases enough for the grad-B drift to become dominant again. The trapped particle then returns to the original precession drift direction at the higher radius where it was transported by the electric field.

In summary, we have observed numerically an $m / n=$ 2/1 MHD mode in LHD which leads to a strong redistribution of perpendicular energetic-particle pressure profile, and is characterized by a rapid frequency chirpingdown in the beginning of the nonlinear phase. It was shown that the helically trapped particles which interact strongly with the mode are pushed in the direction opposite to the classical precession drift direction, and at the 
same time pushed outward. This precession drift reversal is caused by the interaction with the radial electric field of the MHD mode. The electric field creates a strong enough $\mathrm{E} \times \mathrm{B}$-drift to counter the precession drift motion of the particles, and to generate the significant outward motion. Since the precession drift reversal occurs for almost all of the particles which strongly interact with the MHD mode, the associated radial transport causes the significant redistribution of perpendicular energeticparticle pressure profile. Though the MHD mode we have investigated is an $m / n=2 / 1$ mode, the transport process found in this work may occur for the EIC which is an $m / n=1 / 1$ mode and brings about the significant energetic-particle transport in LHD. The experimentally observed $m / n=1 / 1$ EIC was not replicated during our simulations, and more work is needed in order to explore if this phenomenon is important for the EIC. It would be also worth investigating the energetic-particle transport process for the fishbone instability in tokamak plasmas with a special attention to the precession drift reversal.

Numerical computations were performed on the Plasma Simulator (NEC SX-Aurora TSUBASA) of NIFS with the support and under the auspices of the NIFS Collaboration Research program (NIFS20KNST169), the JFRS-1 of the International Fusion Energy Research Centre, and the Supercomputer Fugaku of the RIKEN Center for Computational Science (Project ID: hp210178). This work was supported by MEXT as Program for Promoting Researches on the Supercomputer Fugaku (Exploration of burning plasma confinement physics).

\section{DATA AVAILABILITY}

The data that support the findings of this study are available from the corresponding author upon reasonable request.

${ }^{1}$ K. McGuire, R. Goldston, M. Bell, M. Bitter, K. B. ad K. Brau, D. Bunhenaur, T. Crowley, S. Davis, and F. D. et al., Phys. Rev. Lett. 50, 891 (1983).

${ }^{2}$ L. Chen, R. B. White, and M. N. Rosenbluth, Phys. Rev. Lett. 52, 1122 (1984), URL https://link.aps.org/doi/10. 1103/PhysRevLett.52.1122.

${ }^{3}$ B. Coppi and F. Porcelli, Phys. Rev. Lett. 57, 2272 (1986), URL https://link.aps.org/doi/10.1103/PhysRevLett.57.2272.

${ }^{4}$ M. N. Rosenbluth and P. H. Rutherford, Phys. Rev. Lett. 34, 1428 (1975), URL https://link.aps.org/doi/10.1103/ PhysRevLett.34.1428.

${ }^{5}$ K.-L. Wong, Plasma Physics and Controlled Fusion 41, R1 (1999), URL https://doi.org/10.1088\%2F0741-3335\%2F41\% $2 \mathrm{~F} 1 \% 2 \mathrm{~F} 001$

${ }^{6}$ W. W. Heidbrink, Physics of Plasmas 15, 055501 (2008), https://doi.org/10.1063/1.2838239, URL https://doi.org/10. $1063 / 1.2838239$.

${ }^{7}$ Y. Todo, Rev. Mod. Plasma Phys. 3 (2019), URL https://doi. org/10.1007/s41614-018-0022-9.

${ }^{8}$ Y. Kolesnichenko and V. Oraevskii, At. Energy 23, 1028-1030 (1967).

${ }^{9}$ A. B. Mikhailovskii, AC 68:5 (1975).

${ }^{10}$ L. Chen, Physics of Plasmas 1, 1519 (1994), https://doi.org/10.1063/1.870702, URL https://doi.org/ 10.1063/1.870702.

${ }^{11}$ L. Chen and F. Zonca, Rev. Mod. Phys. 88, 015008 (2016), URL https://link.aps.org/doi/10.1103/RevModPhys.88.015008.
${ }^{12}$ M. Osakabe, S. Yamamoto, K. Toi, Y. Takeiri, S. Sakakibara, K. Nagaoka, K. Tanaka, K. Narihara, and the LHD Experimental Group, Nuclear Fusion 46, S911 (2006), URL https: //doi.org/10. 1088\%2F0029-5515\%2F46\%2F $10 \% 2 \mathrm{Fs} 07$.

${ }^{13} \mathrm{~K}$. Toi, K. Ogawa, M. Isobe, M. Osakabe, D. A. Spong, and Y. Todo, Plasma Physics and Controlled Fusion 53, 024008 (2011), URL https://doi.org/10.1088/0741-3335/53/ 2/024008.

${ }^{14}$ X. D. Du, K. Toi, M. Osakabe, S. Ohdachi, T. Ido, K. Tanaka, M. Yokoyama, M. Yoshinuma, K. Ogawa, K. Y. Watanabe, et al. (LHD Experiment Group), Phys. Rev. Lett. 114, 155003 (2015), URL https://link.aps.org/doi/10.1103/ PhysRevLett.114.155003.

${ }^{15}$ X. Du, K. Toi, S. Ohdachi, M. Osakabe, T. Ido, K. Tanaka, M. Yokoyama, M. Yoshinuma, K. Ogawa, K. Watanabe, et al., Nuclear Fusion 56, 016002 (2015), URL https://doi.org/10. $1088 \% 2 \mathrm{~F} 0029-5515 \% 2 \mathrm{~F} 56 \% 2 \mathrm{~F} 1 \% 2 \mathrm{~F} 016002$.

${ }^{16}$ X. D. Du, K. Toi, S. Ohdachi, K. Y. Watanabe, H. Takahashi, Y. Yoshimura, M. Osakabe, R. Seki, T. Nicolas, H. Tsuchiya, et al. (LHD Experiment Group), Phys. Rev. Lett. 118, 125001 (2017), URL https://link.aps.org/doi/10.1103/ PhysRevLett.118.125001.

${ }^{17}$ T. Bando, S. Ohdachi, M. Isobe, Y. Suzuki, K. Toi, K. Nagaoka, H. Takahashi, R. Seki, X. Du, K. Ogawa, et al., Nuclear Fusion 58, 082025 (2018), URL https ://doi.org/10.1088\% 2F $1741-4326 \% 2 \mathrm{Faac} 699$.

18 J. Varela, D. Spong, L. Garcia, S. Ohdachi, K. Watanabe, and R. Seki, Nuclear Fusion 59, 046008 (2019), URL https://doi. org/10.1088/1741-4326/aaff80.

${ }^{19}$ J. Varela, S. Ohdachi, K. Watanabe, D. Spong, L. Garcia, and R. Seki, Nuclear Fusion 60, 046013 (2020), URL https://doi. org/10.1088/1741-4326/ab7213.

${ }^{20} \mathrm{Y}$. Todo and T. Sato, Physics of Plasmas 5, 1321 (1998), https://doi.org/10.1063/1.872791, URL https://doi.org/10. 1063/1.872791.

${ }^{21}$ Y. Todo, Physics of Plasmas 13, 082503 (2006), https://doi.org/10.1063/1.2234296, URL https://doi.org/ $10.1063 / 1.2234296$.

${ }^{22}$ Y. Todo, M. V. Zeeland, A. Bierwage, and W. Heidbrink, Nuclear Fusion 54, 104012 (2014), URL https ://doi.org/10.1088\% 2 F0029-5515\%2F54\%2F $10 \% 2$ F 104012 .

${ }^{23}$ Y. Todo, M. A. V. Zeeland, A. Bierwage, W. Heidbrink, and M. Austin, Nuclear Fusion 55, 073020 (2015), URL https:// doi . org $/ 10.1088 \% 2 \mathrm{~F} 0029-5515 \% 2 \mathrm{~F} 55 \% 2 \mathrm{~F} 7 \% 2 \mathrm{~F} 073020$.

${ }^{24}$ Y. Todo, M. V. Zeeland, and W. Heidbrink, Nuclear Fusion 56, 112008 (2016), URL https://doi.org/10.1088\%2F0029-5515\% $2 \mathrm{~F} 56 \% 2 \mathrm{~F} 11 \% 2 \mathrm{~F} 112008$.

${ }^{25}$ Y. Todo, New Journal of Physics 18, 115005 (2016), URL https: //doi.org/10.1088\%2F 1367-2630\%2F18\%2F11\%2F115005.

${ }^{26}$ A. Bierwage, K. Shinohara, Y. Todo, N. Aiba, M. Ishikawa, G. Matsunaga, M. Takechi, and M. Yagi, Nature Communications 9, 3282 (2018), URL https://doi.org/10.1038/ s41467-018-05779-0.

${ }^{27}$ Y. Todo, R. Seki, D. A. Spong, H. Wang, Y. Suzuki, S. Yamamoto, N. Nakajima, and M. Osakabe, Physics of Plasmas 24, 081203 (2017), https://doi.org/10.1063/1.4997529, URL https: //doi.org/10.1063/1.4997529.

${ }^{28}$ H. Wang, Y. Todo, T. Ido, and Y. Suzuki, Phys. Rev. Lett. 120, 175001 (2018), URL https://link.aps.org/doi/10.1103/ PhysRevLett.120.175001.

${ }^{29}$ M. Sato and Y. Todo, Journal of Plasma Physics 86, 815860305 (2020).

${ }^{30}$ R. Seki, Y. Todo, Y. Suzuki, K. Ogawa, M. Isobe, D. Spong, and M. Osakabe, Journal of Plasma Physics 86, 815860502 (2020).

${ }^{31}$ K. Harafuji, T. Hayashi, and T. Sato, Journal of Computational Physics 81, 169 (1989), ISSN 0021-9991, URL http://www. sciencedirect.com/science/article/pii/0021999189900697.

${ }^{32}$ Y. Suzuki, N. Nakajima, K. Watanabe, Y. Nakamura, and T. Hayashi, Nuclear Fusion 46, L19 (2006), URL https://doi. org $/ 10.1088 \% 2 \mathrm{~F} 0029-5515 \% 2 \mathrm{~F} 46 \% 2 \mathrm{~F} 11 \% 2 \mathrm{~F} 101$. 
${ }^{33}$ F. Zonca, L. Chen, S. Briguglio, G. Fogaccia, G. Vlad, and X. Wang, New Journal of Physics 17, 013052 (2015), URL https://doi.org/10.1088/1367-2630/17/1/013052. 